\title{
Wind power short-term prediction based on SVM trained by improved
}

\author{
FOA \\ Feng Xiao ${ }^{1, a}$, Guochu Chen ${ }^{1, b}$ \\ ${ }^{1}$ School of Electrical Engineering, Shanghai DianJi University, Shanghai, 200240, China \\ ${ }^{2}$ School of Electrical Engineering, Shanghai DianJi University, Shanghai, 200240, China \\ åCorresponding author:15221856749@163.com \\ ${ }^{b}$ Corresponding author:chengc@sdju.edu.cn
}

\begin{abstract}
Keywords: wind power prediction; prediction accuracy; support vector machine; optimizing; assessment
\end{abstract}

\begin{abstract}
The forecast accuracy of the wind power directly affects the operating cost of the network system, which is directly related to the supply and demand balance grid. Therefore, the forecast accuracy of wind power is very important. Considering the prediction accuracy not high, we propose an improved predictive method that is based on FOA-SVM. Since SVM penalty factor and kernel parameters having a great impact on the forecast Intensive, thus the improved FOA optimizes the parameters of support vector machine and train model with a good parameter optimization .Then the built model is used to the power prediction and evaluates the data finally. The prediction results show: the improved FOA-SVM can produce wind power prediction accuracy better.
\end{abstract}

\section{Introduction}

Due to the uncertainty of wind, randomness, wind power forecasting in large-scale wind farms and network operation plays a key role, in wind farms and network operation, we give an accurate prediction to the wind power .Not only dose it reduce the adverse effects of instability of wind on the grid effectively, but also it provide a strong basis to prepare in advance for power dispatching.

Currently, there are many methods which are applied into wind power forecasting, such as time series analysis ${ }^{[1]}$, BP neural network ${ }^{[2]}$, Kalman filtering method ${ }^{[3]}$, wavelet decomposition ${ }^{[4]}$ and etc. Time series analysis is a data processing method which uses model to analysis and process ordered random data that was observed. However, it will be very different for different models of order. Although BP neural network have good robustness, generalization, fault tolerance, but it is learning convergence slowly and easy to fall into local minimum global and can not get global optimum value. It is difficult for Kalman filtering method to get noise statistics. However, as the youngest of statistical learning theory, support vector machine can learn against small sample data. it also gets good generalization. Since the introduction of kernel function ${ }^{[5]}$, it solves the curse of dimensionality problem effectively. Given the urgency of power prediction and the advantage of support vector machine in the sample regression, this paper established a support vector machine model to forecast short-term power.

Many studies have shown that kernel function parameter $\mathrm{g}$ and penalty factor $\mathrm{c}$ are main factors in support machine kernel which affect the prediction accuracy of SVM. So far, the selection of parameters has no law at all. The randomly selected parameter is less than ideal as demand accuracy. Therefore researchers use the advantages of other algorithms to optimize the parameter of SVM c,g. 
The document ${ }^{[6]}$ proposed a genetic algorithm to optimize the parameter of LS-SVM method and avoid shortage of parameters which is set by people, while reducing the optimization time. The document ${ }^{[7]}$ proposed an optimization parameter based on ant colony algorithm to optimize parameters of SVM. The document ${ }^{[8]}$ proposed support vector machine feature selection and parameter optimization based on bee algorithm. The document ${ }^{[9]}$ proposed face recognition method based on immune algorithm which optimize parameter of $\mathrm{SVM}^{[10]}$ and implement support vector machine parameter being automatically optimized and ultimately accurate classification of facial expression.

At present, Drosophila has not yet been used in wind power prediction which is a new algorithm proposed by Professor Pan Wenchao professor who is in Tai wan in 2011. In this paper, drosophila algorithm is simple, less arguments, less computation, high precision. So it is used to optimize parameter c,g of SVM. And this paper proposed improved optimization algorithm Drosophila which is applied into wind power prediction to improve accuracy.

\section{Improves Drosophila Algorithm And Its Performance Analysis}

\section{Basic drosophila optimization algorithms}

FOA is new method that deduces and seeks global optimization based on the foraging behavior of Drosophila which was proposed by Pan Wenchao who is in Taiwan in 2011. ${ }^{[1]}$

Its optimization step:

(1) initialize randomly Drosophila population position

Init X_axis

Init $\mathrm{Y} \_$axis //X and $\mathrm{Y}$ indicate the position coordinates of Fig. 1(x, y)

(2) Drosophila individual uses olfactory to search direction and distance of food $\mathrm{Xi}=\mathrm{X} \_$axis+Random Value//After random directions and distances and find goals' $\mathrm{X}$ coordinates

$\mathrm{Yi}=\mathrm{Y} \_$axis+Random Value// After random directions and distances and find goals' $\mathrm{Y}$ coordinates

(3) Since we can not know the position of food, we firstly estimate the distance(Dist) between the position and the origin. Then we calculate the flavor concentration determination value $(\mathrm{Si})$.

$$
\begin{gathered}
\text { Disti }=\sqrt{\left(x_{i}^{2}+y_{i}^{2}\right)} \\
S i=1 / \text { Disti }
\end{gathered}
$$

(4) Flavor concentration determination value ( $s$ ) is substituted into the flavor concentration determination function (or called Fitness function). Then we determined flavor concentration (Smelli) of the individual position Drosophila.

$$
\text { Smelli=Function }(\mathrm{Si})
$$

(5) Find the highest concentration fruit fly in the fruit flies.

[bestSmell bestIndex]=max (Smell)

(6) Retain the best flavor concentration and $x$, y coordinates, while Drosophila group flew to the determined position by the visual.

$$
\begin{aligned}
& \text { Smellbest=bestSmell } \\
& \text { X_axis=X (bestIndex) } \\
& \text { Y_axis=Y (bestIndex) }
\end{aligned}
$$

(7) Get into the iterative optimization, repeat steps 2-5, and determine whether the concentration of flavor taste better than the previous iteration concentration. If it is, we 
execute step (6), until we find the optimum flavor concentration.

\section{Improved algorithm Drosophila}

Considering the problem of parameters and premature, we change the population size, the initial poison setting and iteration step to improve Drosophila algorithm's search capability.

Population size is related to the level of search capability, the more flies in search of food, the faster you will find traces of food. Selecting the appropriate amount of the number of fruit fly and setting appropriate initial position can improve problem solving efficiency. While we select a different step value to affect the ability of searching.

Now we analyze the algorithm by solving extreme of function $y=-5+x^{\wedge} 2$. we set the population 3,10,20 flies to search for respectively, and set the number of iterations 10 times, 100 times, 1000 times, and set iterative step value $2 *$ rand ()$-1,20 *$ rand ()$-10$.

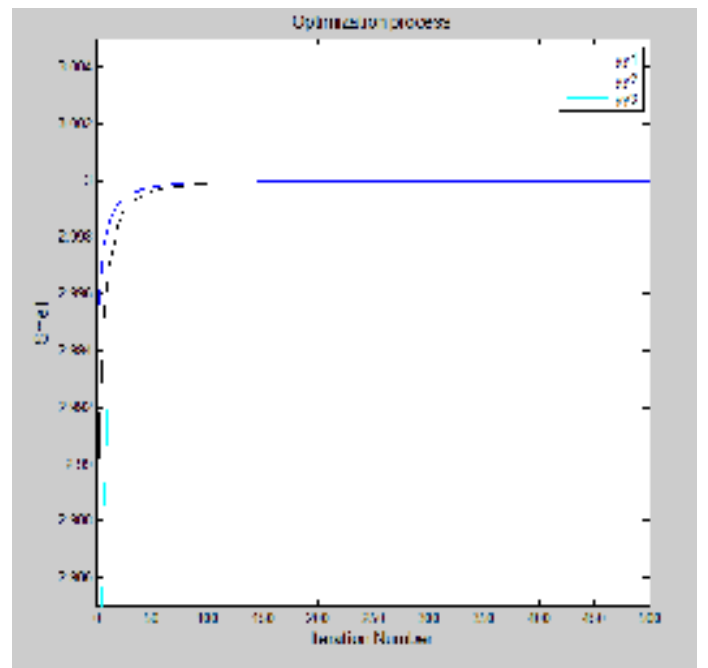

Fig. 1 iterative step value is $2 *$ rand ()$-1$

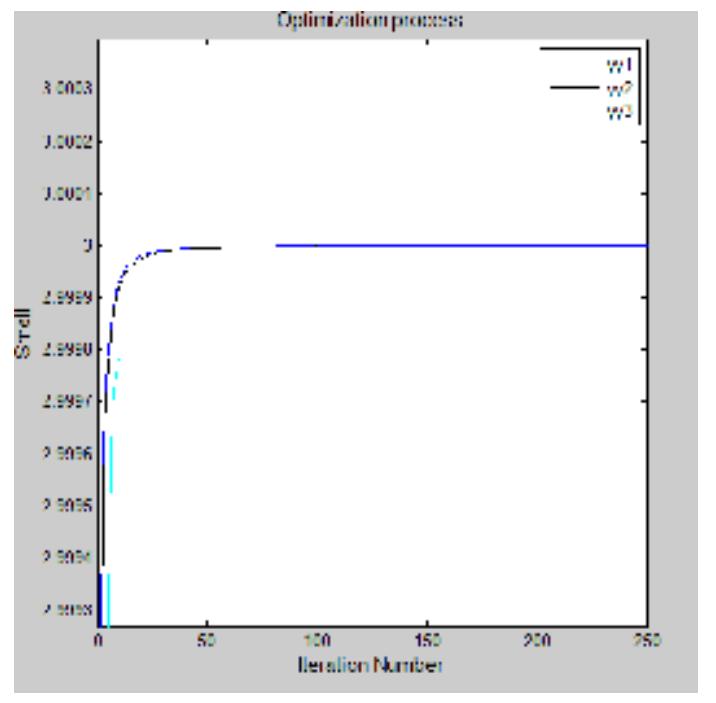

Fig. 2 iterative step value is $20 *$ rand ()$-10$

As can be seen from the fig. 1 and fig.2, it is different for the degree of convergence because of different populations, the number of iterations and iteration step.

\section{Improve convergence of the drosophila}

In the actual calculation, drosophila distance (Dist) gets the random value within a great range. So flavor concentration decision value ( $\mathrm{Si}$ ) may occur in a very small range and it is easy for FOA to fall into local optima and we can not find global extreme. Therefore we add a trip parameter (escape local optima) when we calculate flavor concentration determination value. We change the formula $2-3$ by the parameter.

$$
S_{M}=S_{i}+\Delta ; \quad \Delta=\text { Dist }_{i} \times(0.5-\delta) ; 0 \leq \delta \leq 1
$$

In this way, the determination value Sm expand the distribution. When the Dist is very big, Sm avoid falling into the minimum.

Proof: assume $\mathrm{H}=\{\mathrm{h}(1), \mathrm{h}(2), \mathrm{h}(3), \ldots . \mathrm{h}(\mathrm{t}), \mathrm{h}(\mathrm{t}+1)\}$ the optimal value of each generation from the first generation to $t+1$. So we satisfy

$$
h(1) \leq h(2) \leq \ldots . \leq h(t) \leq h(t+1)
$$

(1) When the flies search for food in a limited range, we can know the optimal solution must converge through the theorem that the number of columns must have the limit if it does not rise and has the lower bound.

(2) When the Dist get the random value in the great range, we adding the parameter $\Delta, \mathrm{Sm}=\mathrm{Si}+\Delta$ will not fall into the local extreme value and will converge to the optimal solution. 


\section{SVM Model Based On Improved Drosophila Algorithm}

\section{Model Establishment}

Firstly, we process and collect the data, select samples and test sets. Then we optimize parameters c,g by using fruit flies algorithm. Secondly, we build the model of prediction to test and analysis forecasting errors. It is shown in fig. 3 .

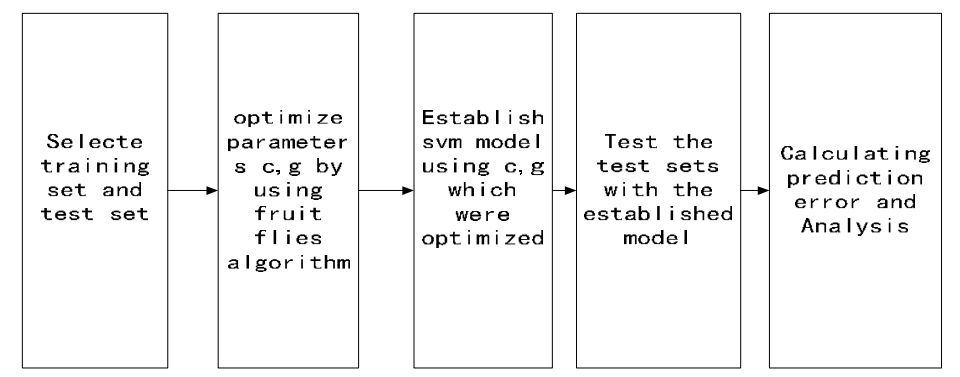

Fig.3 MFOA-SVM model

\section{Choose the best parameter c,g using MFOA}

Drosophila algorithm optimization goal is fitness function. Fitness function in this article is the rms error which is got after predicting data. Fitness value is smaller, the smaller the error. And the prediction rate of SVM regression is better. Specific steps are as follows:

First step: Determine the fitness function that we desired.

Second step: The population size and the number of iterations is initialized in the flies algorithm. Then we choose the SVM parameters.

Third step: We build SVM training model and predict the data, calculate the fitness function. Then we record the best parameter values in each generation group.

Four step: Update Drosophila group position, and repeate the second step until the maximum number of iterations is reached, then we output the optimal value.

Specific flow chart below fig.4:

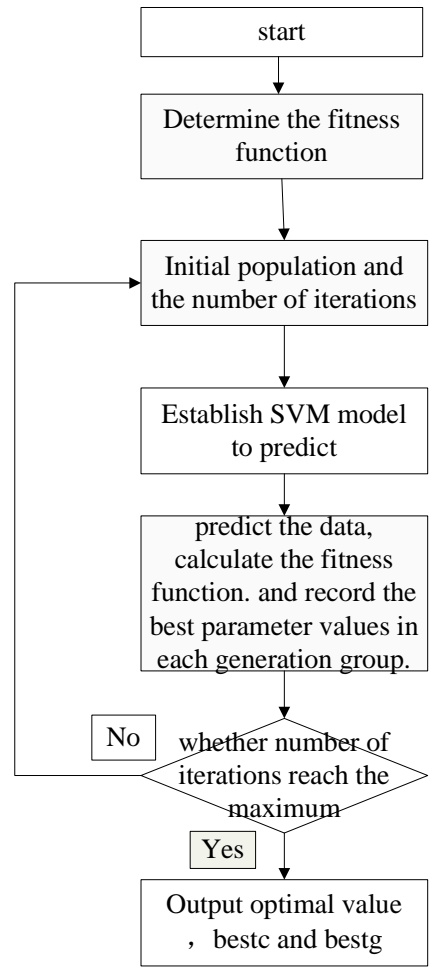

Fig.4 MFOA optimization flow chart 


\section{The Application Of MFOA-SVM In Wind Power Prediction}

Wind power prediction plays an integral role in the overall wind farm. The accuracy of the prediction accuracy will have a direct impact on the supply and demand balance of the grid. And it has a decisive influence on the utilization of wind resource. Thereby increasing the prediction accuracy of power is very important to the entire wind farm. This paper predicts the data of wind farms based on svm power prediction model and optimize the parameters by using drosophila algorithm to improve prediction accuracy.

\section{Data processing}

In this paper, we put the wind farm owning GW82-1500 - km fan as the research object, using MATLAB2009 platform and support vector machine (SVM) toolkit toolbox Libsvm - mat to finish training and prediction of data samples. We use the data which is from the October 10 wind farm wind turbines run of measured data as an example to build sample set. The power value of the wind turbines is divided into training samples(Table1), training objectives(Table2), test samples(Table3) and test objectives(Table4). We put the data that is in a mouth 20 days before as the training sample, the data of later 10 days as the test sample, interception 720 groups of data on October 2 nd day to experience. Selected data is in the fan running stage, but will still be defective machine, fan for a long time for some downtime caused by data more missing time. We take out them directly. For less or no missing data, missing data are only changed data using the following methods to solve:

$$
\text { If } \quad \begin{aligned}
& |P(d, t)-P(d, t-1)|> \\
& \partial * P(d, t-1) \& \&|P(d, t)-P(d, t+1)| \\
& >\partial * P(d, t+1)
\end{aligned}
$$

Then

$$
P(d, t)=[P(d, t-1)+P(d, t+1)] / 2
$$

\begin{tabular}{|c|c|c|c|c|c|}
\hline & (kx) & (kz) & $(k \pi)$ & (kn) & (kn) \\
\hline $0: 00$ & 710.0189 & $732.874:$ & 441.9693 & 245.0219 & 577.892 \\
\hline ::00 & $732.874:$ & 441.9683 & $245.02: 9$ & 577.892 & 156. 6927 \\
\hline $2: 00$ & 441.963 & 245.0219 & 577.892 & 156.6927 & 25.78428 \\
\hline 3:00 & 245.0219 & 577.892 & 156.6927 & 25.78428 & 67.37648 \\
\hline $4: 00$ & 577.892 & 153.6927 & 25.78428 & 67.37346 & 1548963 \\
\hline $5: 00$ & 152. 6927 & 25.78428 & 67.37646 & 1548983 & 398. 0497 \\
\hline 6:00 & 25.78428 & 67.37646 & 1548963 & 389.0497 & 765.575 \\
\hline 7:00 & 67.37646 & 1548983 & 389.0497 & 765.575 & 892. 1917 \\
\hline $8: 00$ & 1548983 & 399.0497 & 765.575 & 892.1947 & 950.681 \\
\hline $9=00$ & 396. 0457 & 765.575 & 892.1947 & 950.681 & 98. 2083 \\
\hline $10: 00$ & 765.575 & 802.1917 & 500.681 & 999.2083 & 1163.498 \\
\hline 11:00 & 802. 1917 & 950. $68:$ & 908.2083 & 1183.499 & 1158.947 \\
\hline $12: 00$ & 950.68: & c25.2083 & 1183.498 & 1158.947 & 1033.062 \\
\hline $13: 00$ & 964. 2063 & 1163. 498 & 1258.947 & 1033062 & 1002.216 \\
\hline $14=00$ & 1163.489 & 1158. 947 & 1033.062 & 1029.216 & 583. 74.8 \\
\hline
\end{tabular}

In the formula (4-2), $P(d, t)$ is the power value of first t time , d day, $P(d, t-1)$ is the value of first $t-1$ time $d$ day values, $P(d, t+1)$ is the value of first $t+1$ times $d$ day, said thresholds.

Table 1 Part of the training samples
Table 2 Part of the training samples

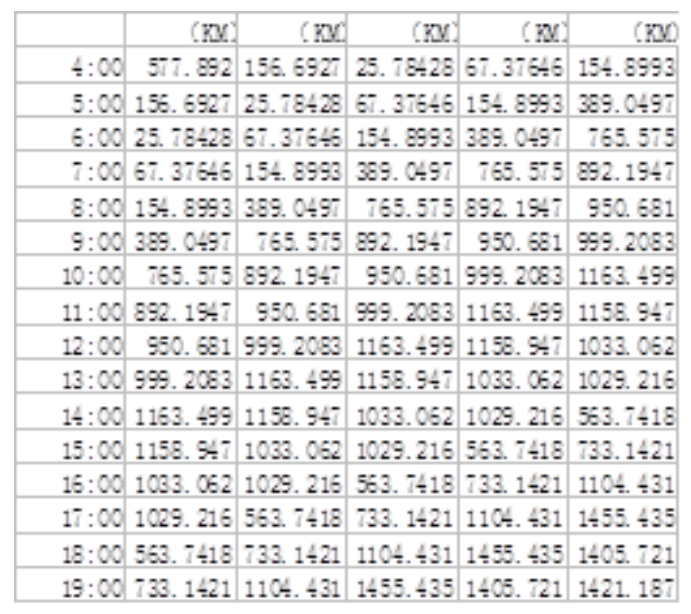


Table 3 Part of the training samples

\begin{tabular}{|c|c|c|c|c|c|}
\hline & (N) & $M$ & KN & (KN) & (Kni) \\
\hline 0.00 & 156.6927 & 25. 78428 & 67. 37646 & 154.8993 & 3) 389.0497 \\
\hline $1: 00$ & 765.575 & 892.1947 & 950.681 & 999.2083 & \begin{tabular}{l|l}
3 & 1163.490
\end{tabular} \\
\hline $2: 00$ & 1158. 94 & 1033. 062 & 1029.216 & 563.7418 & 8 733.1421 \\
\hline $3: 00$ & 1104.431 & 1455.435 & 1405.721 & 1421. 187 & 71120.528 \\
\hline $4: 00$ & 1050.017 & 1260.948 & 1297.926 & 1152.893 & 3 - 1393.26 \\
\hline $5: 00$ & 1164.505 & 1036. 927 & 1352.436 & 803.8626 & .3376 \\
\hline $6: 00$ & 1053. 818 & 1258.976 & 1331.232 & 1477.421 & 11517.728 \\
\hline $7: 00$ & 1512. 906 & 1161. 186 & 584.5951 & $896.67 \div 1$ & 11182.627 \\
\hline $8: 00$ & 1230. 508 & 1017.23 & 1413. 532 & 1376.279 & 564 \\
\hline $9: 00$ & 63978 & 35. $8952=$ & $=90.80847$ & 126.3859 & 418 \\
\hline $10: 0$ & 560.9613 & 235.7495 & 81.6828 & 56.63972 & $219 \div .516$ \\
\hline $11: 00$ & 78. @215 & 77.28573 & 13. 52231 & 60.96592 & 2138.226 \\
\hline $12: 00$ & 86. 38577 & 72. 04039 & 252.1552 & 566.2648 & 6 548.501 . \\
\hline $13: 00$ & 757.1951 & 754.8292 & 786.1591 & 386.035 & 5441.74 \\
\hline$x, \infty$ & 00 & & & & \\
\hline
\end{tabular}

Table 4 Part of the training samples

\begin{tabular}{|l|l|l|l|l|l|l|l|l|l|l}
1399.412 & 1396.222 & 1408.518 & 1443.788 & 1351.051
\end{tabular} 1472.21407 .683800 .6431570 .3044131 .2985

\section{Experimental process}

According to 3.1 figure 3 for model establishment and prediction, parameter selection shall be carried out in accordance with the flow chart of figure 4, choose RBF kernel function, the model of specific training steps are as follows:

(1) Eliminate, transform and add data collected

(2) classify data and the normalized processing

(3) initialize population size and the number of iterations and train the SVM model to determine the optimal parameter $\mathrm{c}, \mathrm{g}$

(4) We put the processed data into the SVM model to predict

(5) According to the fitness function of what we need, we calculate the fitness value of each generation and record the best value

(6) Through the comparison of each iteration, it is concluded that the optimal value. we output the prediction accuracy.

\section{Results analysis of experiment}

We divide data provided into training samples and testing samples, and put the data normalize processing. Through the FOA - training SVM model, get the kernel function parameters: the bestg $=0.1687$, bestc $=3.3740$, using the obtained parameters test and the normalized processing, the output data as shown in figure 5 and figure 6 

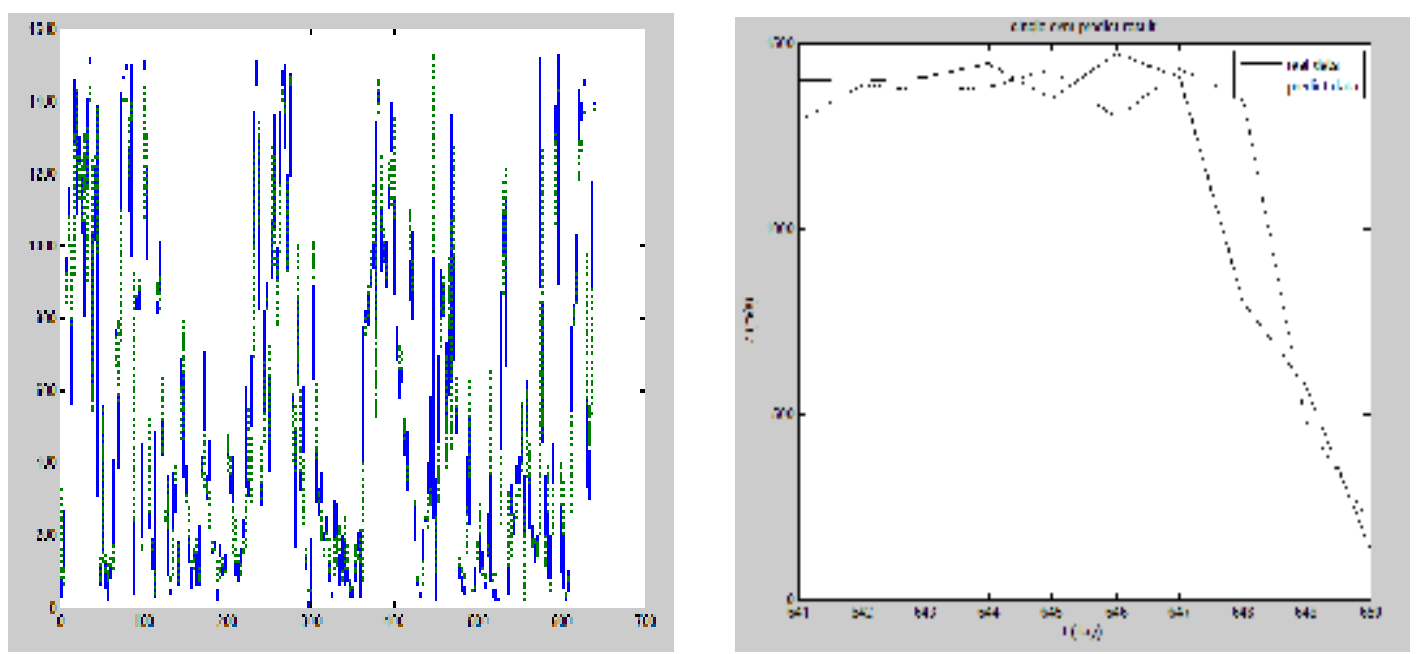

Fig.5 original data and optimized data of test sample Fig.6 original data and optimized data of test goal

From figure 5 and figure 6 we can see through the improved MFOA - SVM optimization that prediction effect is remarkable by changing the population, the number of iterations and iteration step value, continuous experiment. Finally the average relative errors (in fig.7) can achieves 0.1429 , compared to the error 0.1948 of not using MFOA optimization significantly increased, and the correlation is from 0.68 to 0.84 . Thus prediction accuracy is improved.

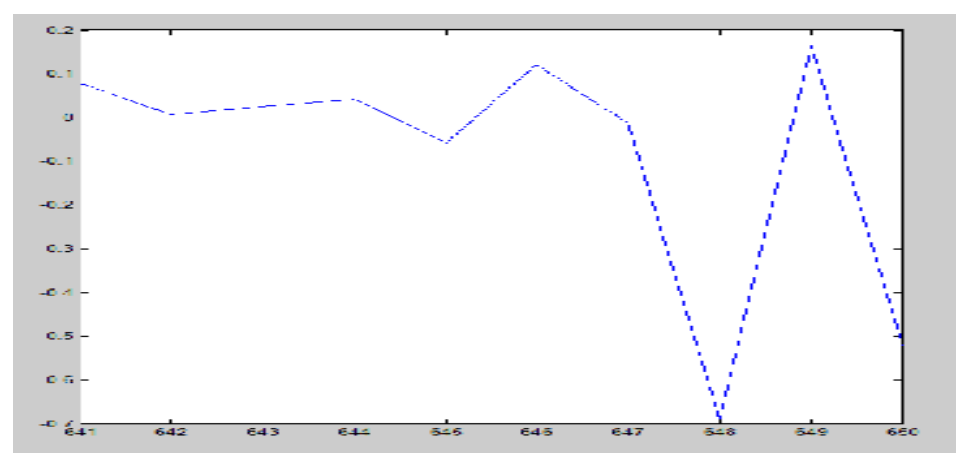

Fig.7 test target prediction after the average relative error

\section{Conclusions}

Wind power prediction is very important for the resources utilization. Accurate forecast is good for power system dispatching departments to adjust scheduling plan timely. Meanwhile, it can reduce the influence of instability of wind power grid. Based on the experimental analysis we adopt an improved algorithm of fruit flies to optimize the parameters of the SVM and build prediction model based on MFOA-SVM to improve the precision of prediction. The simulation results show that fruit flies as a relatively new optimization algorithm used in wind power prediction also shows a very good side.

\section{Acknowledgements}

This work was financially supported by scientific research innovation projects of Shanghai municipal education commission (Grant No.13YZ140) and the key disciplines of Shanghai Municipal Education Commission of China (Grant No.J51901). 


\section{References}

[1] Yang Mao, Sun Chong, Mu Gang, etc. Multiple sampling scale wind power based on time series multi-step prediction research [J]. Electric measurement and instrumentation, 2014, 51 (23): $55-58$

[2] Jiang-ping Yang. Wind speed and power of short-term prediction based on neural network combination forecast [D]. Chongqing: Chong Qing University, 2012(In Chinese)

[3] Zhao Pan, Yiping Dai, Xia Junrong, etc., short-term power wind based on kalman filter prediction model correction [J]. Journal of xi 'an Jiao tong university, 2011, (5): 48-51(In Chinese)

[4] Huang Jensheng. Wind power prediction based on wavelet analysis and support vector machine (SVM) [D]. Shandong University, 2012(In Chinese)

[5] Chi-Hung Wu,Gwo-Hshiung Tzeng. A Novel hybrid genetic algorithm for kernel function and parameter optimization in support vector regression [J].Expert Systems with Applications, 2009, (36):4725-4735.

[6]Keqi Wang, shaochun Yang, Tianhong Dai, Xuebing Bai. Using genetic algorithm to optimize parameters of least squares support vector machine (SVM) method [J]. Journal of computer applications and software, 2009, 26 (7) : 109-111

[7]Zhuan Yan, Bai Zhenlin, Xu Yunfeng. Support vector machine (SVM) based on ant colony algorithm [J]. Journal of computer simulation, 2011, 28 (5) : 216-219

[8] Chen Yuan, Ma Hongwei. Support vector machines feature selection and parameters optimization based on the bees algorithm [J]. Journal of combination machine tools and automatic processing technology, 2013, 11:41-43

[9] Tian Yantao, Liu Shuaishi, Wan Chuan, Chen hw, Li Dong, Wang Xinzhu, Sui Zhen. Optimize parameters of facial expression recognition method of support vector machine (SVM) based on the improved immune algorithm [P]. China invention patent, 101968853 a. 2010-10-15

[10] Lei Dong, Xiaozhong Liao, Wang Lijie. Large wind farm power modeling and prediction [M]. Beijing: science press, 2014-39(In Chinese)

[11] Wenchao Pan. Flies optimization algorithm [M]. 2011(In Chinese) 